\title{
Lattice Kaon Physics
}

\author{
P A Boyle*† \\ School of Physics \\ SUPA \\ $J C M B$ \\ University of Edinburgh \\ Edinburgh UK EH9 3JZ \\ E-mail: paboyle@ph.ed.ac.uk
}

I review lattice quantum chromodynamics as relevant to the kaon system. Topics covered include the pseudoscalar masses and decay constants, the chiral effective lagrangian, $f_{K} / f_{\pi}$, semi-leptonic kaon decay form factors, and the neutral kaon oscillation parameter $B_{K}$.

2009 KAON International Conference KAON09,

June 09 - 122009

Tsukuba, Japan

\footnotetext{
* Speaker.

${ }^{\dagger}$ University of Edinburgh
} 
This paper reviews the status of Lattice QCD where relevant to kaon physics. Some summary intended for non-experts of the broader status of the field is given in section 1. I interpret this goal as including some aspects of pion physics and the Chiral Lagrangian in section 2.

I review the status of Lattice QCD input to determining $V_{u s}$, section 3, both via $f_{K} / f_{\pi}$, section 3.1, and $K_{l 3}$ section 3.2. The kaon bag parameter $B_{K}$ which contributes to the $\varepsilon_{K}$ constraint on the unitarity triangle is reviewed, section 4 . Lattice $K-\pi \pi$ calculations were reviewed at this conference by Norman Christ [1] and are thus beyond the scope of this review.

Carrying out a review of this nature is an increasingly daunting task in the presence of the excellent Flavia Lattice Averaging Group effort [2]. It is also worth mentioning excellent recent reviews by individual members of FLAG [3,4]. In many areas it is difficult to match this comprehensive effort. My approach therefore is to try to highlight theoretical issues, distinguishing features, and methods of the lattice calculations that are not easily conveyed by the compressed asterisk rating system; I do not necessarily attempt the comprehensive categorisation and averaging task this working group has undertaken except where I may have significant updates from new material, or wish to differ in the analysis.

$\mathrm{PAB}$ wishes to thank the many authors whose original research has been reviewed and emphasizes that any use of Lattice world averages or quoted results herein must cite the orginal source(s) where the intellectual value was created.

\section{Current Large Scale Lattice Simulations}

Despite many recent advances in both algorithms and computing hardware Lattice QCD cannot presently be performed at physical masses in a large volume for reasons of numerical cost. Were this feasible, there would be little debate about methods in the field. In practice, the choice of approach, table 1, is a complex optimisation problem where the selected approach is dependent on the physical processes for which one aims to yield a minimal overall error. These errors are composed of statistical and finite volume, mass extrapolation and discretisation systematics. Strategy is influenced by estimates of the importance \& calculability of finite volume, NNLO chiral expansion effects, and even the influence of strange quark loops.

The physics goal of computing chirally structured weak matrix elements ( $B_{K}$ and $K \rightarrow \pi \pi$, for example) makes perhaps the biggest rationally justifiable difference of approach. RBC-UKQCD and JLQCD use substantially more expensive methods to obtain near exact chiral symmetry and gain access to richer phenomenology than would otherwise be possible. These approaches also give automatic $O(a)$ improvement off-shell, making improved RI-mom non-perturbative renormalisation simpler. However, less theoretically pristine approaches are substantially cheaper and BMW and MILC in particular have used this to gain access to finer lattice spacings and lighter simulated masses in large volumes.

Lattice QCD gives complete freedom in choice of the quark masses (dynamical loops and valence legs) in numerical simulation. Purely unitary simulations maintain the valence quark masses equal to those of simulated dynamical flavors in all matrix elements. A partially quenched simulation is a superset of the corresponding unitary simulation; here the multiple valence masses are used (both equal to and not equal to the dynamical masses) and next-to-leading order PQ- $\chi^{\mathrm{PT}}$ is 


\begin{tabular}{c|c|c|c|c|c|c|c} 
Collaboration & Action & $\mathrm{L}(\mathrm{fm})$ & $m_{\pi}^{\min }(\mathrm{MeV})$ & $a^{-1} \mathrm{GeV}$ & $\mathrm{ChPT}$ & Unitary & FV corr. \\
\hline JLQCD[5] & Overlap & 1.7 & 310 & 1.8 & $\mathrm{SU}(2)$ & $\mathrm{U}$ & $\mathrm{CDH}$ \\
RBC-UKQCD [6] & DWF & $2.0,2.7$ & 330 & $1.7,2.3$ & $\mathrm{SU}(2), \mathrm{SU}(3)$ & $\mathrm{PQ}$ & - \\
ETMC [7] & Twisted mass & 2.0 & 300 & $1.9,2.2,2.8$ & $\mathrm{SU}(2)$ & $\mathrm{U}$ & $\mathrm{CDH}$ \\
PACS-CS[8] & Clover & 3.1 & 156 & 2.2 & $\mathrm{SU}(2), \mathrm{SU}(3)$ & $\mathrm{U}$ & $\mathrm{CDH}$ \\
BMW [15] & Clover & 4.0 & 190 & $1.6,2.3,3.03$ & polynomial & - & - \\
MILC[16] & Staggered & $2.4,2.9$ & 320 & $1.1,1.3,1.6$ & rs-SU(3) & PQ & -
\end{tabular}

Table 1: Summary of the parameters of major lattice calculations reviewed

available for a variety of important quantities such as pseudoscalar masses, decay constants and the kaon bag parameter.

RBC-UKQCD and MILC have used partially quenched analyses to increase the information within the chiral regime, while JLQCD, ETMC, BMW and PACS-CS used unitary simulations.

Where smaller volumes are used, it is common for finite volume corrections to be applied to the data prior to chiral extrapolation. This, of course, takes the effect of finite volumes as an input to (rather than output of) the lattice calculation. The presently favoured model is the resummed scheme of Colangelo, Durr and Haefeli $(\mathrm{CDH})$ and is applicable only to unitary datapoints, and cannot be combined with partially quenched analyses. For this reason the calculations where $m_{\pi} L$ becomes rather smaller than around 3.2 both apply finite volume correction and consider only unitary datapoints.

Approaches to mass extrapolations include the application of SU(3) and SU(2) chiral effective theories. BMW recently adopted Taylor expansion having achieved both light simulation masses and a large volume. They feel sufficiently close to the physical point to include polynomial fits in their analysis; these are viewed as an analytic expansion around the non-zero physical quark mass in a massive region whose validity does not extend to the chiral non-analyticity at zero.

\subsection{Is lattice QCD ab initio in practice?}

Lattice QCD has rightly enjoyed the reputation of an ab initio method to solve QCD. However, present analysis \& extrapolation approaches do involve varying degrees of non-lattice input. What is clear is that, in some cases, the extrapolated results have relinquished the significant benefit of having only the QCD Lagrangian as input.

Ideally, any use of the low energy expansion of QCD for chiral extrapolation should be performed in a consistent \& well motivated context. Either complete NLO or complete NNLO should be used; it should both describe the data and appear a well convergent series within the range of the data without ad hoc resummation schemes or partial NNLO where only favoured analytic terms are included, or certain LEC's biased towards phenomenological input.

Taking the long view, lattice QCD remains systematically improvable. Continued advance in supercomputing power will soon remove the need for such extrapolations, just as the quenched approximation has been long discarded. However, it is worth highlighting mass extrapolation and finite volume issues as areas where the field should do better. 


\section{Chiral Effective Lagrangian}

The chiral effective theory can be viewed in two roles by a lattice practitioner. First it can be viewed as an important predictive tool which can be verified and understood with lattice QCD (i.e. intrinsic interest). The chiral effective theory has also been used as a critical component of making well founded mass extrapolations from simulation in almost all recent lattice calculations (i.e. practical value). The former requires that chiral perturbation theory be a well convergent series at the relevant physical masses, while the latter requires convergence above the physical masses.

Were SU(3) chiral effective theory convergent at the kaon mass, it would be the natural framework for interpreting lattice data. MILC in particular have relied on the partially quenched SU(3) approach to remove unitarity violating discretisation effects introduced by their "rooting" prescription [17]. However, some determinations of the leading order low energy constant $F_{0}$ representing the pseudo-scalar decay constant in the SU(3) chiral limit (table 2) have been surprisingly low.

RBC-UKQCD found [6] that NLO SU(3) formula does not describe pseudoscalar masses and decay constants above $m_{\pi} \simeq 400 \mathrm{MeV}$. In addition to surprisingly low results for the LEC, RBCUKQCD obtained a slightly low result $f_{\pi}=124.1(3.6)(6.9) \mathrm{MeV}$; with a single lattice spacing this is within systematic error of the physical value. At simulated masses and even at the pion mass the SU(3) correction to leading order was surprisingly large, figure 1. For this reason, they introduced the application of $S U(2) \chi^{\mathrm{PT}}$ to both pionic and kaonic quantities in an underlying $2+1$ flavor simulation $[11,6]$, and this has been adopted by other groups.

In this $\mathrm{SU}(2)$ effective theory the kaon is treated as a heavy external meson field $[11,12,6]$. The least convergent terms expand in $\left(\frac{m \pi}{m_{K}}\right)^{2}$, and this appears the more convergent approach for current data than $S U(3)$ expansion where the least convergent terms expand in $\left(\frac{m_{\eta}}{4 \pi f}\right)^{2}$.

The PACS-CS collaboration also found that kaon data was better described by SU(2)[13, 14], figure 2; however their result for $F_{0}$ was much larger and the conclusion about simulated 200$400 \mathrm{MeV}$ pions differs. The underlying data for $f_{\pi}$ is qualitatively similar between PACS-CS and RBC-UKQCD but differs by around $4 \%$. PACS-CS determine $Z_{A}$ from one loop lattice perturbation theory; whether both the overall scale and curvature of their chiral fits would become closer with a non-perturbative $Z_{A}$ remains an open question.

The MILC collaboration found a larger $F_{0}$ than RBC-UKQCD [16]; however this is not necessarily discrepant when one considers that the RBC-UKQCD results were from a single lattice spacing. The SU(3) chiral limit could be clarified relatively easily by simulations with artificially light "strange" quarks..

\subsection{The curious linearity of lattice data}

BMW have not yet published a detailed comparison of their analytic fits to their chiral fits [15]. However several collaborations have found suprising linearity in their data for $f_{\pi}$.

Since the Kaon conference RBC-UKQCD have presented a second lattice spacing [18, 19] at Lattice 2009, where they found good scaling compared with their earlier calculation and form conclusions about the continuum limit with theoretically pure dynamical chiral fermions. They set the scale using the $\Omega$ baryon. Figure 1 demonstrates that while a simple linear from their 290 $450 \mathrm{MeV}$ simulated masses produces the PDG $f_{\pi}$, NLO extrapolation does not. The discrepancy is 


\begin{tabular}{lll}
\hline Collaboration & $F_{0}(\mathrm{MeV})$ & $F / F_{0}$ \\
\hline MILC [16] & $106(8)$ & $1.15(5)\left(_{-3}^{+13}\right)$ \\
RBC/UKQCD [6] & $93.5(7.3)$ & $1.229(59)$ \\
PACS-CS[8] & $118.5(9)$ & $1.078(58)$ \\
\hline
\end{tabular}

Table 2: There remains some spread in recent results for the leading order low energy constant $F_{0}$ of the SU(3) chiral effective theory. A low value would imply SU(3) corrections for kaons in particular must be uncomfortably large. $F$ is the $\mathrm{SU}(2)$ low energy constant and here the normalisation is such that the pion decay constant is around $131 \mathrm{MeV}$.

consistent with a naturally sized NNLO effect; however the cancellation to produce linearity within statistical error is striking. This data corroborates the BMW claim that Taylor expansion in the region directly above the physical masses shows good convergence; however the presence of known non-analytic terms makes it difficult to continue analytic fits to lighter masses and RBC-UKQCD used the difference between analytic and chiral fits as a systematic error in predictions[18].
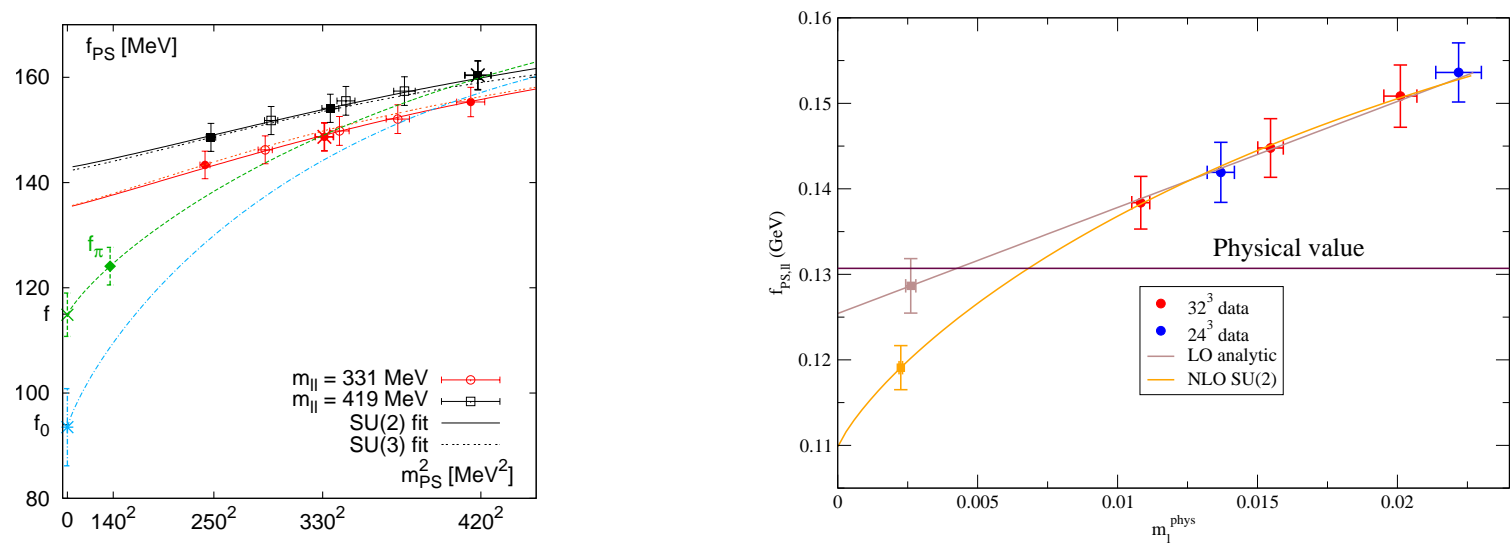

Figure 1: Left: NLO chiral extrapolation of RBC-UKQCD's dynamical DWF decay constant for $f_{\pi}$ on their $1.73 \mathrm{GeV}$ ensemble. The decay constant in the SU(3) limit is surprisingly low, and corrections in this expansion are uncomfortably large. This plot displays partially quenched data. Right: Since the Kaon conference RBC-UKQCD have presented a preliminary continuum limit for their simulations. The data points here are adjusted to represent values in the continuum limit and compared to both an NLO and linear extrapolation. The scale is taken from $M_{\Omega}$, and NLO $\chi^{\mathrm{PT}}$ yields a pion decay constant that is inconsistent with its physical value, while linear extrapolation is in agreement. The inconsistency is certainly explicable by a naturally sized NNLO effect, however the coincidence of cancellation between orders to leave such linear behaviour at this level of precision is curious. This plot displays only the unitary datapoints for simplicity.

\subsection{Low energy constants}

Lattice determinations of the leading order and next-to-leading order LEC's have been surveyed in detail by S. Necco, and by FLAG in excellent recent reviews [4, 2]. I reproduce here some of the SU(2) NLO summary in table 3, as I believe this is more convergent and hence more meaningful than SU(3). I recommend arXiv:0901.4257 [4] for details and further results, including a survey of the large body of $\varepsilon$-regime results which are not covered in this paper. 

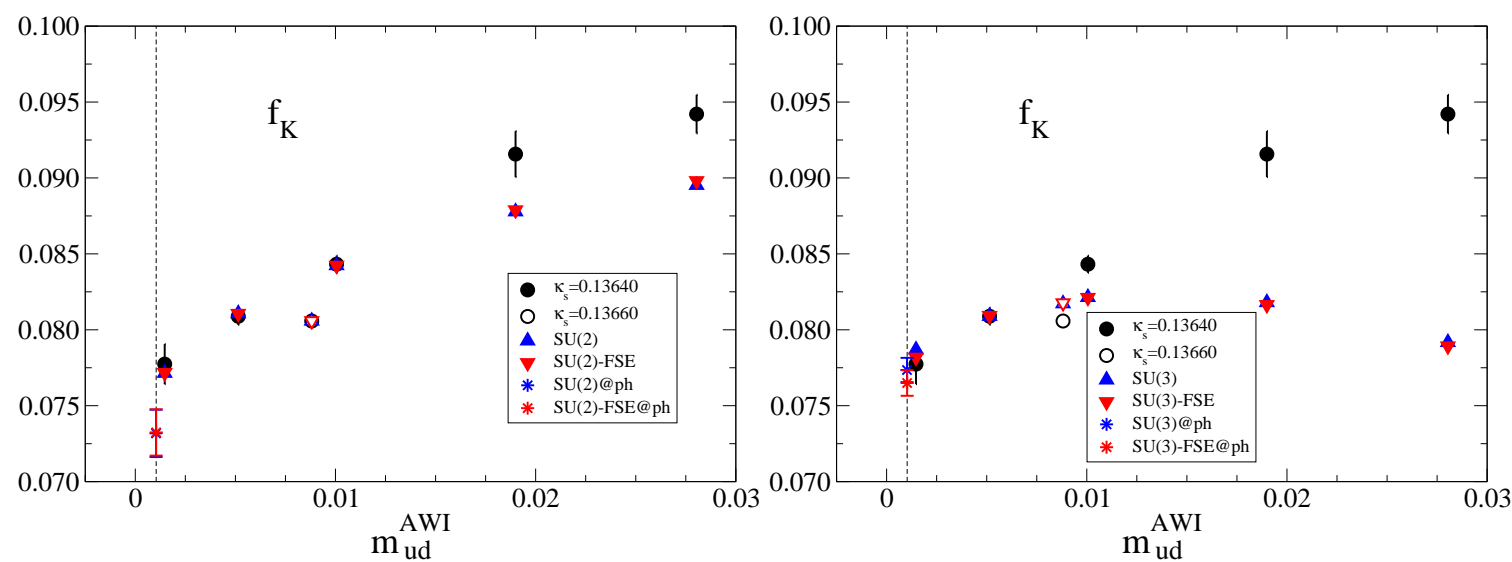

Figure 2: PACS-CS NLO fits to the kaon decay constant using SU(2) (left) and SU(3) (right) $\chi^{\mathrm{PT}}$. As with RBC-UKQCD SU(2) is found to give better agreement at larger masses. The vertical dashed line represents the physical point, albeit in a small volume. The "FSE" fits are corrected for finite size effects using CDH. SCALE?

\begin{tabular}{|c|c|c|c|c|c|c|}
\hline Collaboration & \multicolumn{2}{|r|}{$N_{f}$} & $F(\mathrm{MeV})$ & $F_{0}(\mathrm{MeV})$ & $\Sigma^{1 / 3}(\mathrm{MeV})$ & $\Sigma_{0}^{1 / 3}(\mathrm{MeV})$ \\
\hline \multicolumn{2}{|c|}{ ETM [20] } & 2 & \multicolumn{2}{|c|}{$86.03(5)$} & \multicolumn{2}{|l|}{$267(2)(9)(4)$} \\
\hline \multicolumn{2}{|c|}{ JLQCD/TWQCD[21] } & 2 & \multicolumn{2}{|c|}{$79.0(2.5)(0.7)\left(\begin{array}{l}+4.2 \\
-0.0\end{array}\right)$} & \multicolumn{2}{|l|}{$235.7(5.0)(2.0)\left(\begin{array}{l}+12.7 \\
-0.0\end{array}\right)$} \\
\hline MILC[23] & & $2+1$ & & & $278(1)\left({ }_{-3}^{+2}\right)(5)$ & $242(9)\left(\begin{array}{c}+5 \\
-17\end{array}\right)(4)$ \\
\hline RBC/UKQCD[6] & & $2+1$ & $81.2(2.9)(5.7)$ & & $255(8)(8)(13)$ & \\
\hline PACS-CS[8] & \multicolumn{2}{|r|}{$2+1$} & $90.3(3.6)$ & $83.8(6.4)$ & $309(7)$ & $290(15)$ \\
\hline ETM[24] & \multicolumn{2}{|r|}{2} & $86.6(4)(7)$ & & $264(2)(5)$ & \\
\hline Collaboration & 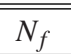 & & (SU(2)) & $\overline{\bar{l}_{4}(\mathrm{SU}(2))}$ & $\bar{l}_{3}(\mathrm{SU}(3))$ & $\overline{\bar{l}_{4}(\mathrm{SU}(3))}$ \\
\hline ETM & 2 & & $42(8)(10)(27)$ & $4.59(4)(2)(13)$ & & \\
\hline ETM & 2 & & $2(4)(2)$ & $4.4(1)(1)$ & & \\
\hline JLQCD/TWQCD & 2 & & $44(57)\left(\begin{array}{c}+0 \\
-68\end{array}\right)\left(\begin{array}{c}+32 \\
-0\end{array}\right)$ & $4.14(26)\left(\begin{array}{c}+49 \\
-0\end{array}\right)($ & & \\
\hline MILC & $2+1$ & & & & $1.1(6)\left(\begin{array}{l}+1.0 \\
-1.5\end{array}\right)$ & $4.4(4)\left(\begin{array}{l}+4 \\
-1\end{array}\right)$ \\
\hline RBC/UKQCD & $2+1$ & & $13(33)(24)$ & $4.43(14)(77)$ & $2.87(28)$ & $4.10(5)$ \\
\hline PACS-CS & $2+1$ & & $14(23)$ & $4.04(19)$ & $3.47(11)$ & $4.21(11)$ \\
\hline
\end{tabular}

Table 3: (Abridged) summary tables from [4], which also reviews SU(3) LEC's and the large body of work making use of the $\varepsilon$-regime. Here $\mathrm{F}$ is in the convention where $f_{\pi} \simeq 92 \mathrm{MeV}$

\subsection{Pion form factors and $l_{6}$}

The pion vector form factor is related to the low energy constant $l_{6}$ :

$$
\begin{gathered}
\left\langle\pi^{+}\left(p^{\prime}\right)\left|V_{\mu}\right| \pi^{+}(p)\right\rangle=F^{\pi \pi}\left(q^{2}\right)\left(p_{\mu}+p_{\mu}^{\prime}\right) \\
\left\langle r_{\pi}^{2}\right\rangle=\left.6 \frac{d}{d q^{2}} f^{\pi \pi}\left(q^{2}\right)\right|_{q^{2}=0}=-\frac{12 l_{6}^{r}}{f^{2}}-\frac{1}{8 \pi^{2} f^{2}}\left(\log \frac{m_{\pi}^{2}}{\mu^{2}}+1\right) .
\end{gathered}
$$

A summary of recent results for the pion charge radius is given in table 4. There are presently considerable discrepancies in the results at intermediate masses prior to chiral extrapolation, and consequently differing conclusions. 
ETMC and JLQCD [24-27] obtain somewhat lower values than RBC-UKQCD and QCDSF in the region $300 \leq m_{\pi} \leq 500 \mathrm{MeV}$, and conclude the NNLO effects in the chiral expansion are significant. This calculation typically involves the use of a number discrete Fourier modes for $q$ with model dependent interpolation used to obtain the derivative at $q^{2}=0$. RBC-UKQCD and ETMC $[30,24]$ obtained good resolution in the low $q^{2}$ region, figure 3. Here, a twisted boundary condition with phase $e^{i \theta}$ applied to valence Fermions on the simulated torus allows to vary lattice momenta smoothly between the standard periodic and anti-periodic Fourier modes, enabling several momenta between the zero and first Fourier modes [28]. RBC-UKQCD found that NLO $\chi^{\mathrm{PT}}$ yielded good results for the pion charge radius, but this remains a puzzle however, since ETMC also used twisted boundary conditions but concluded NNLO $\chi^{\mathrm{PT}}$ was required.

The determination of the pion scalar charge radius by JLQCD is worth noting and which has been performed including the effects of disconnected quark flow diagrams for the first time[26].

\begin{tabular}{c|c|c|c} 
Collab & Action & $\left\langle r_{\pi}^{2}\right\rangle_{V}$ & $\left\langle r_{\pi}^{2}\right\rangle_{S}$ \\
\hline ETMC [24] & 2f TM & $0.456(38)$ & \\
JLQCD [25, 26] & 2f Overlap & $0.409(23)(37)$ & $0.617(79)(66)$ \\
JLQCD [27] & 2f Clover & $0.396(10)$ & $0.60(15)$ \\
QCDSF [29] & 2f Clover & $0.441(19)$ & \\
\hline RBC-UKQCD[30] & 2+1f DWF & $0.418(31)$ & \\
LHPC [31] & 2+1f MILC/DWF & $0.310(46)$ &
\end{tabular}

Table 4: Summary of recent results for the pion charge radius from lattice QCD
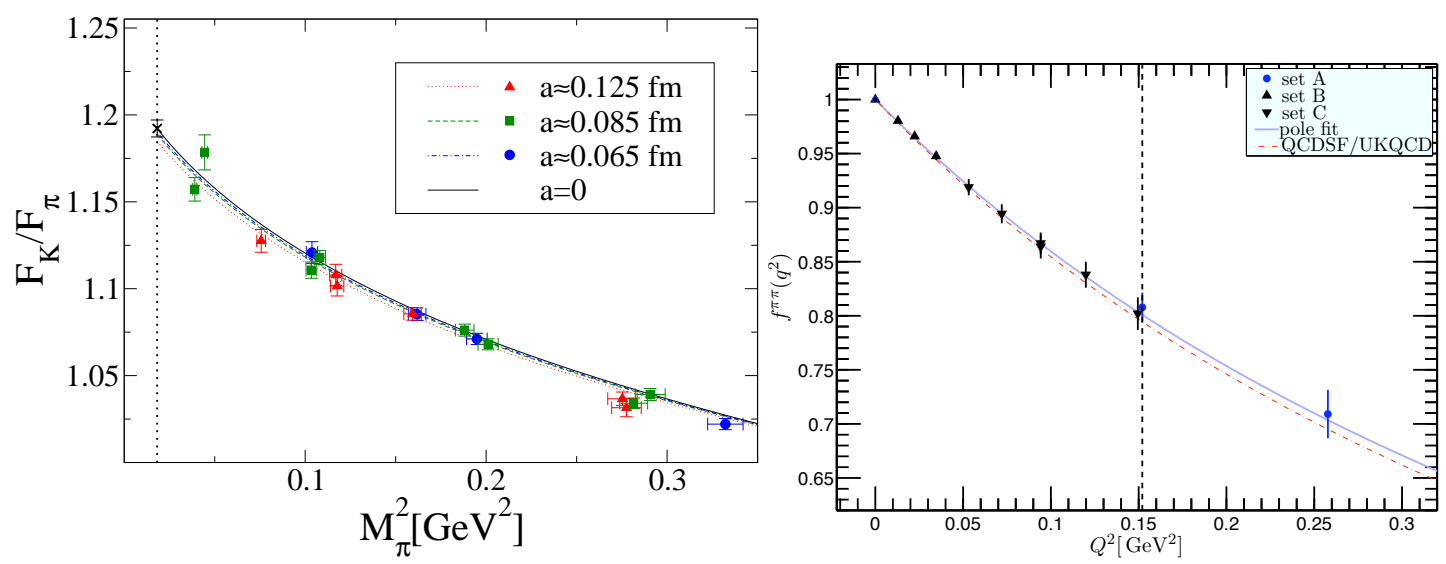

Figure 3: LEFT: BMW's chiral extrapolation of $f_{K} / f_{\pi}$ [15]. This includes an impressive $190 \mathrm{MeV}$ lightest simulated mass, $4 \mathrm{fm}$ volumes and a continuum limit. Detailed exposition in print is absent, but nevertheless this is an exciting calculation for which further details are eagerly awaited. RIGHT: RBC-UKQCD mapped out the deep infra-red region of the pion form factor using twisted boundary conditions. ETMC used a similar approach down $0.05 \mathrm{GeV}^{2}[30]$

\section{$2.4 L_{10}^{r}$}

Here we see the first of many quantities that are made accessible with chirally symmetric lattice actions. $L_{10}^{r}$ is related to the electromagnetic component of the splitting between $\pi^{+}$and $\pi^{0}$, and is determined from small difference between the axial and vector vacuum polarisation functions. 


$$
\begin{aligned}
\Pi_{V_{\mu v}}-\Pi_{A_{\mu \nu}} & =\left(q^{2} \delta_{\mu v}-q_{\mu} q_{v}\right) \Pi_{V-A}^{(1)}-q_{\mu} q_{v} \Pi_{V-A}^{(0)} \\
\Pi_{V-A}^{(1)} & =-\frac{f_{\pi}^{2}}{q^{2}}-8 L_{10}^{r}(\mu)-\frac{\log \left(\frac{m_{\pi}^{2}}{\mu^{2}}+\frac{1}{3}-H(x)\right)}{24 \pi^{2}}
\end{aligned}
$$

JLQCD produced a beautiful first determination in their $n_{f}=2$ overlap simulation [32]. obtaining the pion e-m mass squared splitting (in chiral limit; c.f. PDG with physical $u, d$ masses $1261 \mathrm{MeV}^{2}$ ):

$$
\begin{gathered}
L_{10}^{r}\left(m_{\rho}\right)=-5.2(2)_{-3}^{+5} \times 10^{-3} \\
m_{\pi^{ \pm}}^{2}-m_{\pi^{0}}^{2}=993(12)\left(_{-135}^{+0}\right)(149) \mathrm{MeV}^{2}
\end{gathered}
$$

A second calculation has been performed by RBC-UKQCD with $2+1$ fomain wall fermions [33]. The calculation is also interesting in that it produces information about the radius of convergence of $\chi^{\mathrm{PT}}$ in momentum space, in addition to its convergence in pion mass; these are similar.

3. $V_{u s}$

The two best lattice constraints for $V_{u s}$ involve SU(3) breaking effects: for $\frac{f_{K}}{f_{\pi}}$ this is $\mathrm{O}(20 \%)$, and for $f_{0}^{K \pi}\left(q^{2}=0\right)$ this is $\mathrm{O}(4 \%)$. While the $K_{l 3}$ approach is less mature, it looks very promising and is rapidly becoming better studied. We consider these two quantities in turn.

\section{1 $V_{u s}$ from $f_{K} / f_{\pi}$}

This topic was reviewed in detail by Lellouch [3]. The calculation with the smallest quoted errors is the mixed action HISQ/Asqtad staggered calculation by HPQCD[35], followed by the smeared clover simulation from BMW[15].

The HPQCD calculation used a mixed action. While published in an abbreviated form, it appears to have not followed MILC's best practice of performing a partially quenched rs- $\chi^{\mathrm{PT}}$ analysis to absorb unitarity violations. Rather, it appears to have matched the HISQ valence pseudoscalar Goldstone taste in to the (irrelevant here) AsqTad valence pseudoscalar Goldstone taste. This differs from the various relevant masses of sea pseudoscalar multiplet. It is hard for lattice theorists not directly involved in the calculation for form a clear judgement with the available information.

Figure 3 (left) reproduces the truly impressive BMW simulation that spans three lattice spacings and includes volumes up to $4 \mathrm{fm}$ and pion masses down to an impressive $190 \mathrm{MeV}$. The BMW calculation has not yet received a detailed exposition in print, and has so far lacked a nonperturbative determination of the axial current renormalisation so important checks on $f_{K}$ and $f_{\pi}$ seperately have not yet been performed; it seems likely non-perturbative operator improvement was not used and resulted in their dual use of $a$ and $a^{2}$ continuum extrapolation. Their chiral extrapolation was interesting but details are sparse; chiral expansion and Taylor expansion fits were combined weighted by quality of fit and only the combined fit has been shown. Publication of the qualities of fit, and direct comparison of the two in the style of figure 1, would be interesting since continuing an analytic fit downwards is not without risk and it is not yet clear how much the lightest data points constrain chiral curvature. 
Since the Lellouch review, ETMC have updated [36] their $N_{f}=2$ twisted mass result to $f_{K} / f_{\pi}=1.210(18)$. As this will not substantially change the lattice average I recommend continued use of that quoted by Lellouch [3] while noting the leading publications are in formats that are not amenable to critical assessment

$$
f_{K} / f_{\pi}=1.194(3)(10)
$$

\section{2 $V_{u s}$ from $K_{l 3}$}

The semi-leptonic form factor

$$
\left\langle\pi\left(p^{\prime}\right)\left|V_{\mu}\right| K(p)\right\rangle=f_{+}\left(q^{2}\right)\left(p_{\mu}+p_{\mu}^{\prime}\right)+f_{-}\left(q^{2}\right)\left(p_{\mu}-p_{\mu}^{\prime}\right)
$$

can be computed precisely using several "double ratios" following [37, 38], such as

$$
\frac{\left\langle K(\overrightarrow{0})\left|V_{0}\right| \pi(\overrightarrow{0})\right\rangle\left\langle K(\overrightarrow{0})\left|V_{0}\right| \pi(\overrightarrow{0})\right\rangle}{\left\langle K(\overrightarrow{0})\left|V_{0}\right| K(\overrightarrow{0})\right\rangle\left\langle\pi(\overrightarrow{0})\left|V_{0}\right| \pi(\overrightarrow{0})\right\rangle}=\frac{\left(m_{K}+m_{\pi}\right)^{2}}{4 m_{K} m_{\pi}}\left|f_{0}\left(q_{\max }^{2}\right)\right|^{2}
$$

\begin{tabular}{lllcccc}
\hline \hline Ref. & $N_{f}$ & action & $a[\mathrm{fm}]$ & $L[\mathrm{fm}]$ & $\begin{array}{c}M_{\pi}^{\min }[\mathrm{MeV}] \\
\text { typ/val }\end{array}$ & $f_{+}(0)$ \\
\hline JLQCD [40] & 2 & NP SW & 0.09 & 1.8 & $550 / 550$ & $0.967(6)$ \\
RBC[41] & 2 & DWF & 0.12 & 2.5 & $490 / 490$ & $0.968(9)(6)$ \\
ETMC [42] & 2 & tmQCD & 0.11 & 2.7 & $260 / 260$ & $0.9581(57)(35)$ \\
FNAL/MILC[44] & $2+1$ & KS+Wil & & & & $0.962(6)(9)$ \\
RBC/UKQCD[43] & $2+1$ & DWF & 0.11 & $1.8,2.8$ & $290 / 240$ & $0.9644(33)(34)(14)$ \\
\hline \hline
\end{tabular}

Table 5: An update of the corresponding table by Lellouch [3] to include new ETMC results
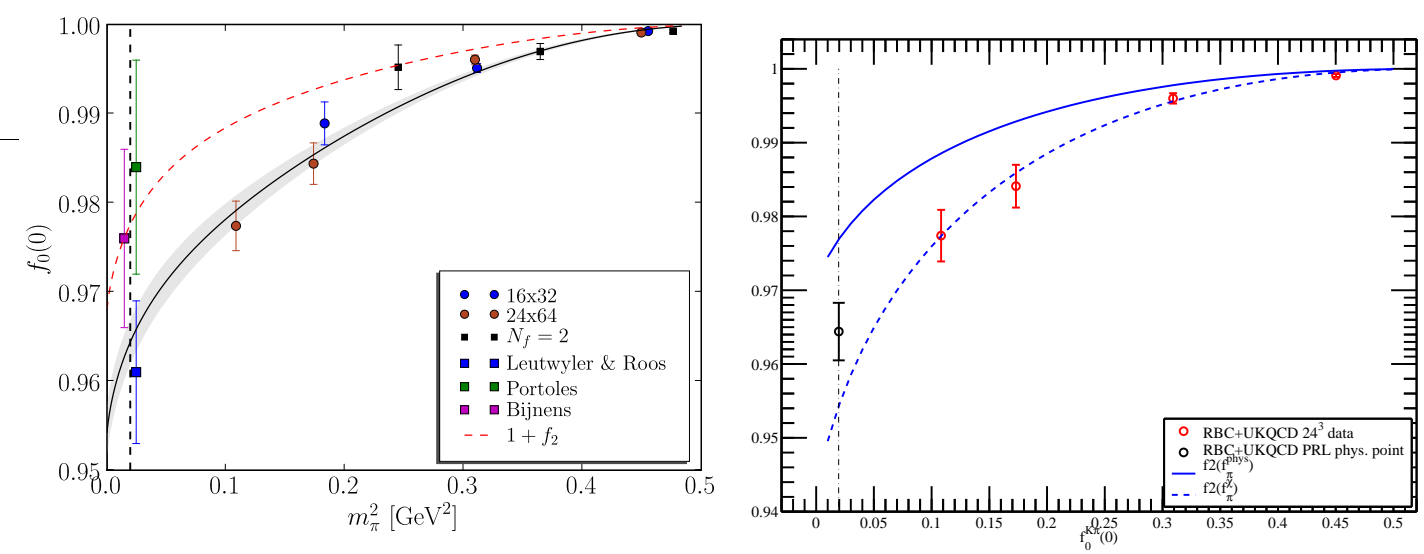

Figure 4: LEFT: RBC-UKQCD chiral extrapolation of $f_{+}(0)$ represents the best lattice constraint and has $2+1$ flavors. RIGHT: Courtesy A. Jüttner - using RBC-UKQCD's result for $F_{0}$ to better define $f_{2}$ results in NLO describing the data well and the chiral expansion of this small breaking looking naturally convergent. A redefined $\Delta_{f}$ would be near zero. 
The form factor $f_{+}\left(q^{2}=0\right)$ is of key interest for $V_{u s}$ and has chiral expansion $1+f_{2}+f_{4} \ldots$ where $f_{n} \simeq O\left(M_{K, \pi}^{n} /\left(4 \pi F_{0}\right)^{n}\right)$. The best constraint in, table 5 uses $2+1$ flavors and is from RBCUKQCD [43]. Their chiral extrapolation is shown in figure 4 obtaining

$$
f_{+}(0)=0.9644(33)^{\text {stat }}(34)^{\text {extrapolation }}(14)^{\text {disc }} \text {. }
$$

The calculation used Fourier modes, with model dependence in $q^{2}$ interpolation and chiral extrapolation; there was no controlled continuum limit and a discretisation systematic error was budgeted at $4 \%$ of $1-f_{+}(0)$. The historical use of $f_{\pi}$ as the denominator for $f_{2}$ is not the only sensible choice. Taking the above RBC-UKQCD result for $F_{0}$ in table 2 better matches the data and rearranges the expansion at NNLO and above. Different reasonable choices of $f_{2}$ seem to be an important systematic in SU(3) based extrapolations of the form factor. RBC-UKQCD presented additional data at the lattice conference making use of twisted boundary conditions to simulate directly at $q^{2}=0[39,45]$ and have developed an SU(2) formalism [46] which they will use in the chiral extrapolation of their new data.

The ETMC $n_{f}=2$ calculation [42] had a notably robust chiral extrapolation with pion masses as low as $260 \mathrm{MeV}$ and made use of both SU(3) and SU(2) formalisms in their chiral extrapolation. An adjustment was made (but not quoted here) to "correct" the lattice calculation for the missing strange quark using the $\mathrm{SU}(3)$ expression for $f_{2}$. The degree to which leading effects of the absent strange loops are already reabsorbed when the lattice spacing is determined makes this unconvincing; I prefer to quote the unadjusted ETMC calculation as an excellent $n_{f}=2$ result.

4. $B_{K}$

The matrix element

$$
B_{K}=\frac{\left\langle K^{0}\left|\mathscr{O}_{\mathrm{VV}+\mathrm{AA}}\right| \bar{K}^{0}\right\rangle}{\frac{8}{3}\left\langle K^{0} \mid A_{0}\right\rangle\left\langle A_{0} \mid K^{0}\right\rangle}
$$

is multiplicatively renormalised in the continuum and for lattice actions with chiral symmetry. Nonsymmetric actions must deal with unphysical taste/chirality mixings which inflate errors. In some cases mixed actions are used (e.g. ALV, ETMC), where a valence quark action with multiplicative operator renormalisation is combined with a cheaper action for sea quarks; resultant unitarity violations should be fitted away, typically using mixed action chiral perturbation theory.

\section{1 $B_{K}$ in the quenched approximation}

For many years the benchmark quenched $B_{K}$ calculation was from JLQCD using many lattice spacings with the staggered fermion action [47]. This calculation involved the lattice perturbative treatment of a taste mixed operator basis. The continuum limit of

$$
B_{K}^{\overline{\mathrm{MS}}}\left(n_{f}=0,2 \mathrm{GeV}\right)=0.565(4)(5),
$$

obtained by JLQCD [48] using the chirally symmetric domain wall fermion formulation, gives continuum limit consistent with various earlier calculations. This includes RBC who used a different gauge action thus displaying universality. JLQCD's results are particularly clean in that they apply non-perturbative step scaling to enable matching to continuum perturbation theory at large scales. 
This is substantially below the previous quenched "benchmark", and resolved a puzzle highlighted by Lellouch. It also highlights that lattice renormalisation issues of various formulations can be a very important effect.
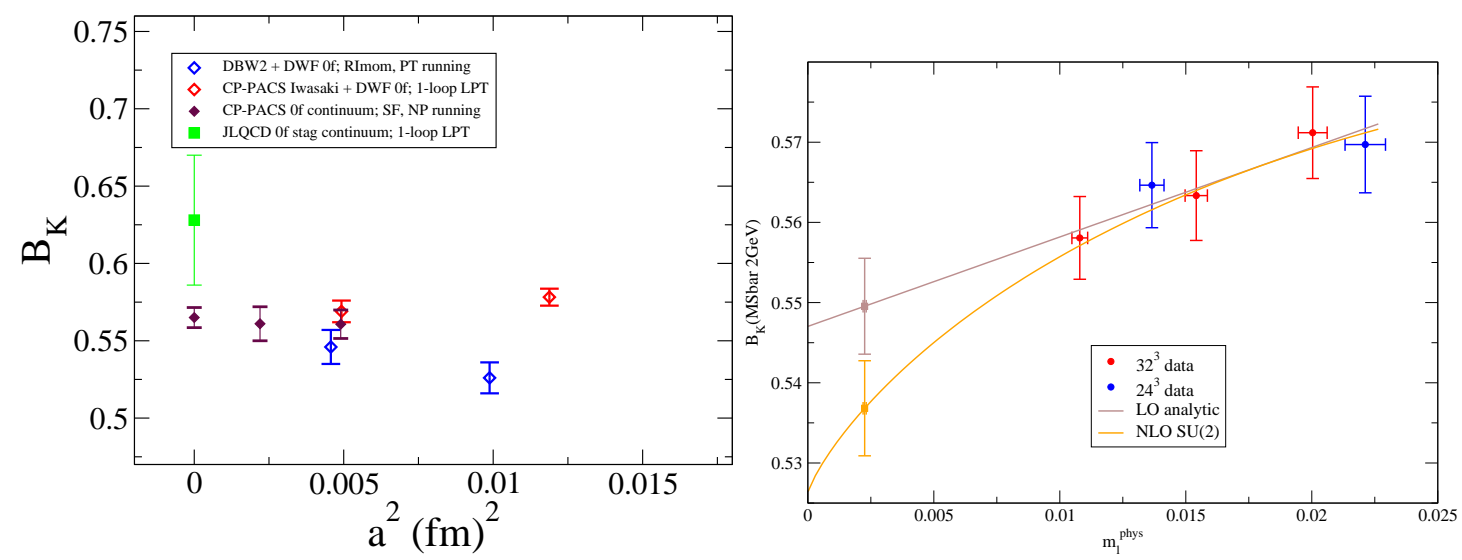

Figure 5: LEFT: Quenched continuum limit of $B_{K}$ has been substantially clarified, with the previous benchmark using staggered fermions appearing suspect. The chirally symmetric DWF formulation has only multiplicative renormalisation and CP-PACS have taken a high quality continuum limit with non-perturbative renormalisation. This is plausibly consistent with both RBC (non-perturbative) and earlier (perturbatively renormalised) CP-PACS results. RIGHT: RBC-UKQCD chiral extrapolation of $B_{K}$ in the continuum limit; data points are shifted to the continuum limit and overlayed. Systematic error taken from difference between linear and SU(2) NLO fits.

\section{$4.22 \mathrm{f} B_{K}$}

There are two recent and high quality determinations of $B_{K}$ in the two flavour theory. JLQCD [49] made an impressive calculation with $2 \mathrm{f}$ overlap action, while ETMC used a somewhat less aesthetic mixed action approach with Osterwalder-Seiler valence fermions with $2 \mathrm{f}$ twisted mass sea fermions [50]. JLQCD obtained $B_{K}^{\overline{\mathrm{MS}}}\left(n_{f}=2,2 \mathrm{GeV}\right)=0.537(4)(40)$ and ETMC obtained $B_{K}^{\overline{\mathrm{MS}}}\left(n_{f}=2,2 \mathrm{GeV}\right)=0.56(2)$ both with single lattice spacings. The ETMC calculaton is preliminary and has not yet addressed important unitarity violation systematics and I quote:

$$
B_{K}^{\overline{\mathrm{MS}}}\left(n_{f}=2,2 \mathrm{GeV}\right)=0.537(4)(40) .
$$

\section{$4.32+1 f B_{K}$}

Two 2+1f calculations were considered in detail at the Kaon conference: RBC-UKQCD's dynamical domain wall simulation, and the Aubin/Laiho/Van de Water mixed DWF valence and staggered (MILC) sea calculation. RBC-UKQCD's programme had previously published results from a single lattice spacing $[6,11]$, and this result had been combined by Lellouch with the 2+1f staggered result of Gamiz et al. [51] in his world average. I choose not to include this staggered result in an average in light of the quenched discrepancy above. The RBC calculation had a preliminary update (since the Kaon conference) at Lattice 2009 with a second lattice spacing and a preliminary joint chiral and continuum extrapolation. These results are sufficiently significant 
that they merit inclusion in these proceedings. This year Aubin, Laiho and Van de Water [52] used a mixed action approach with valence domain wall fermions on $2+1 \mathrm{f}$ of staggered sea quarks.

A detailed comparison of both calculations is interesting in that estimates of systematic errors are in fact the dominant source of uncertainty. The statistical errors in both calculations are subleading and it is worth emphasizing the subjective nature of the systematic error estimates that dominate the quoted errors. The most important differences arise from the estimate of the renormalisation and chiral-continuum extrapolation errors.

ALV base their error on the non-perturbative renormalisation on the difference from mean field improved 1-loop lattice perturbation theory. However, RBC-UKQCD now use multiple nonexceptional momentum renormalisation schemes to gain control over their error estimate.

Given figure 1, RBC-UKQCD estimated its chiral-continuum extrapolation error based on the difference between linear and SU(2) NLO extrapolation, figure 5. ALV apply SU(3), which is in principle less convergent than $\mathrm{SU}(2)$, and estimate the corresponding error based on varying a subset of analytic NNLO terms included in the fit. They do not include taste violation effects beyond NLO or non-analytic terms beyond NLO. It is likely that the difference here lies in the approaches to error estimation.

The RBC-UKQCD result, however, is a preliminary conference submission and has not yet been given an estimate of finite volume effects, nor been published as even a preprint. Until these systematic errors have been published in peer reviewed journals I recommend continued use of [11]

\begin{tabular}{cccc}
\multicolumn{4}{c}{$B_{K}^{\overline{\mathrm{MS}}}\left(n_{f}=2+1,2 \mathrm{GeV}\right)=0.524(30)$} \\
\\
$\mathrm{ALV}$ & $\mathrm{RBC}-\mathrm{UKQCD}(\mathrm{Lat} 09)$ & $\mathrm{RBC}-\mathrm{UKQCD}(2007)$ \\
\hline$B_{K}^{\mathrm{MS}}\left(n_{f}=2+1,2 \mathrm{GeV}\right)$ & $0.527(6)(20)$ & $0.537(19)$ & $0.524(10)(28)$ \\
\hline $\mathrm{NP}$ renormalisation & $3.3 \%$ & $2.4 \%$ & $2 \%$ \\
Chiral/cont extrapolation & $1.9 \%$ & $2.4 \%$ & $2 \%, 4 \%$ \\
statistical & $1.2 \%$ & $1.1 \%$ & $1.9 \%$ \\
Scale \& masses & $0.8 \%$ & $0 \%$ & $1 \%$ \\
Finite volume & $0.6 \%$ & - & $1 \%$ \\
\hline total (quad) & $4.0 \%$ & $3.5 \%$ & $5.7 \%$
\end{tabular}

Table 6: Leading calculations of $B_{K}$ with $2+1 \mathrm{f}$. Systematic error estimates are dominant, and a detailed breakdown of the sources error useful. RBC-UKQCD use a more convergent SU(2) approach to $\chi^{\mathrm{PT}}$ while ALV absorb taste mixings of heavy pseudoscalars with $\mathrm{SU}(3) \chi^{\mathrm{PT}}$.

\subsection{Renormalisation of $B_{K}$}

The dominant systematic error in $B_{K}$ has become the QCD renormalisation. The four recent high quality $2 \mathrm{f}$ and $2+1 \mathrm{f}$ simulations $[49,50,11,52,18]$ all make use of the Rome-Southamptom RI-mom scheme to match between lattice and the $\overline{M S}$ regularisation schemes via an intermediate physically defined MOM scheme.

By historical accident, the particular kinematic point selected was an exceptional momentum point, and suffered avoidably from [53] strong non-perturbative contamination. This paper demon- 
strated that a better choice is to use non-exceptional momenta as the intermediate MOM scheme, and preliminary results have been presented at the lattice conference using a 1-loop calculation by Sachrajda and Sturm. In fact, multiple ostensibly equivalent 1-loop schemes were used and the spread gave a much more robust estimate of the systematic error at around $2.4 \%$, leaving it as the (jointly) dominant source of error.

While other components of the lattice determination of $B_{K}$ will continue the rapid progress seen recently, the determination of this matching to two loops at this non-exceptional momentum point is required for reduction of the lattice error on $B_{K}$ to below around $2 \%$.

The non-exceptional renormalisation point is also even more important to the robust determination of the matrix elements of supersymmetry induced four quark operators[34].

\section{Conclusions}

These are truly exciting times for lattice QCD as simulations push steadily closer to the physical point in a large volume and in the continuum limit. There has been tremendous recent progress in a number of important areas; understanding of the chiral expansion of QCD has been greatly helped by Lattice QCD; Lattice QCD input to $V_{u s}$ is hugely important and the kaon bag parameter has been substantially better determined.

The kaon is the ideal system for Lattice QCD; it contains rich and non-trivial CP violating physics at energy scales that can be simulated without large cut-off effects. Nascent work on electromagnetic and iso-spin breaking effects was beyond the scope of this review; however, it appears that there are no barriers to the continued improvement in the precision of lattice simulations beyond those posed by computing power.

\section{Acknowldgements}

PAB is funded by RCUK fellowship, and wishes to warmly thank both KEK and the CCS at the University of Tsukuba for both support and hospitality.

\section{References}

[1] N. H. Christ, in proceedings of Kaon 09, POS (KAON0 9) 027.

[2] G. Colangelo, in proceedings of Kaon 09, POS (KAONO9) 029.

[3] L. Lellouch, Kaon physics: a lattice perspective, [arXiv:0902.4545 [hep-lat]]

[4] S. Necco, Chiral low-energy constants from lattice QCD, POS (CONF INEMENT 08) 024 [arXiv:0901.4257 [hep-lat]].

[5] T. Kaneko et al. [JLQCD Collaboration], JLQCD's dynamical overlap project, PoS LAT2006 (2006) 054 [arXiv:hep-lat/0610036].

[6] C. Allton et al. [RBC-UKQCD Collaboration], Physical Results from 2+1 Flavor Domain Wall QCD and SU(2) Chiral Perturbation Theory, Phys. Rev. D 78 (2008) 114509 [arXiv:0804.0473 [hep-lat]].

[7] B. Blossier et al. [European Twisted Mass Collaboration], Light quark masses and pseudoscalar decay constants from $N f=2$ Lattice QCD with twisted mass fermions, JHEP 0804 (2008) 020 [arXiv:0709.4574 [hep-lat]]. 
[8] S. Aoki et al. [PACS-CS Collaboration], 2+1 Flavor Lattice QCD toward the Physical Point, arXiv:0807.1661 [hep-lat].

[9] L. Giusti and M. Luscher, Chiral symmetry breaking and the Banks-Casher relation in lattice QCD with Wilson quarks, JHEP 0903 (2009) 013 [arXiv:0812.3638 [hep-lat]].

[10] R. Frezzotti, V. Lubicz and S. Simula, Electromagnetic form factor of the pion from twisted-mass lattice $Q C D$ at $N f=2$, arXiv:0812.4042 [hep-lat].

[11] D. J. Antonio et al. [RBC Collaboration and UKQCD Collaboration], Neutral kaon mixing from 2+1 flavor domain wall QCD, Phys. Rev. Lett. 100 (2008) 032001 [arXiv:hep-ph/0702042].

[12] J. Gasser, C. Haefeli, M. A. Ivanov and M. Schmid, Integrating out strange quarks in ChPT, Phys. Lett. B 652 (2007) 21 [arXiv:0706.0955 [hep-ph]].

[13] Y. Kuramashi, PACS-CS results for 2+1 flavor lattice QCD simulation on and off the physical point, arXiv:0811.2630 [hep-lat].

[14] D. Kadoh et al. [PACS-CS Collaboration], SU(2) and SU(3) chiral perturbation theory analyses on meson and baryon masses in 2+1 flavor lattice QCD, arXiv:0810.0351 [hep-lat].

[15] S. Durr et al., Ab-Initio Determination of Light Hadron Masses, Science 322 (2008) 1224 [arXiv:0906.3599 [hep-lat]].

[16] A. Bazavov et al., Full nonperturbative QCD simulations with 2+1 flavors of improved staggered quarks, arXiv:0903.3598 [hep-lat].

[17] C. Aubin and C. Bernard, Staggered chiral perturbation theory, Nucl. Phys. Proc. Suppl. 129 (2004) 182 [arXiv:hep-lat/0308036].

[18] C. Kelly et. al., in proceedings of Lattice 09 POS (LAT2009) 087.

[19] R. D. Mawhinney et. al., in proceedings of Lattice 09 POS (LAT2009) 081.

[20] P. Dimopoulos, R. Frezzotti, G. Herdoiza, K. Jansen, C. Michael and C. Urbach [ETM Collaboration], Scaling and chiral extrapolation of pion mass and decay constant with maximally twisted mass QCD, arXiv:0810.2873 [hep-lat].

[21] J. Noaki et al. [JLQCD and TWQCD Collaborations], Convergence of the chiral expansion in two-flavor lattice QCD, Phys. Rev. Lett. 101 (2008) 202004 [arXiv:0806.0894 [hep-lat]].

[22] C. Aubin et al. [MILC Collaboration], Light pseudoscalar decay constants, quark masses, and low energy constants from three-flavor lattice QCD, Phys. Rev. D 70 (2004) 114501 [arXiv:hep-lat/0407028].

[23] C. Bernard et al., Status of the MILC light pseudoscalar meson project, PoS LAT2007 (2007) 090 [arXiv:0710.1118 [hep-lat]].

[24] R. Frezzotti, V. Lubicz and S. Simula, Electromagnetic form factor of the pion from twisted-mass lattice QCD at $N f=2$, arXiv:0812.4042 [hep-lat].

[25] T. Kaneko et al. [JLQCD Collaboration and TWQCD collaboration], Pion vector and scalar form factors with dynamical overlap quarks, arXiv:0810.2590 [hep-lat].

[26] S. Aoki et al. [JLQCD Collaboration and TWQCD Collaboration], Pion form factors from two-flavor lattice QCD with exact chiral symmetry, arXiv:0905.2465 [hep-lat].

[27] S. Hashimoto et al. [JLQCD Collaboration], Pion form factors in two-flavor QCD, PoS LAT2005 (2006) 336 [arXiv:hep-lat/0510085]. 
[28] P. A. Boyle, J. M. Flynn, A. Juttner, C. T. Sachrajda and J. M. Zanotti, "Hadronic form factors in lattice QCD at small and vanishing momentum transfer," JHEP 0705 (2007) 016 [arXiv:hep-lat/0703005].

[29] D. Brommel et al. [QCDSF/UKQCD Collaboration], The pion form factor from lattice QCD with two dynamical flavours, Eur. Phys. J. C 51 (2007) 335 [arXiv:hep-lat/0608021].

[30] P. A. Boyle et al., The pion's electromagnetic form factor at small momentum transfer in full lattice QCD, JHEP 0807 (2008) 112 [arXiv:0804.3971 [hep-lat]].

[31] F. D. R. Bonnet, R. G. Edwards, G. T. Fleming, R. Lewis and D. G. Richards [Lattice Hadron Physics Collaboration], Lattice computations of the pion form factor, Phys. Rev. D 72 (2005) 054506 [arXiv:hep-lat/0411028].

[32] E. Shintani et al. [JLQCD Collaboration], S-parameter and pseudo-Nambu-Goldstone boson mass from lattice QCD, Phys. Rev. Lett. 101 (2008) 242001 [arXiv:0806.4222 [hep-lat]].

[33] J. Wennekers et. al., in proceedings of Lattice 09 POS (LAT2009) 074.

[34] J. Wennekers et. al., in proceedings of Lattice 2008 PoS (LATTICE2008) 074.

[35] E. Follana, C. T. H. Davies, G. P. Lepage and J. Shigemitsu [HPQCD Collaboration and UKQCD Collaboration], High Precision determination of the pi, $K, D$ and $D_{\text {s }}$ decay constants from lattice QCD, Phys. Rev. Lett. 100 (2008) 062002 [arXiv:0706.1726 [hep-lat]].

[36] B. Blossier et al., Pseudoscalar decay constants of kaon and D-mesons from Nf=2 twisted mass Lattice QCD, JHEP 0907 (2009) 043 [arXiv:0904.0954 [hep-lat]].

[37] D. Becirevic et al., The $K->$ pi vector form factor at zero momentum transfer on the lattice, Nucl. Phys. B 705 (2005) 339 [arXiv:hep-ph/0403217].

[38] S. Hashimoto, A. X. El-Khadra, A. S. Kronfeld, P. B. Mackenzie, S. M. Ryan and J. N. Simone, Lattice QCD calculation of anti-B -> D l anti-nu decay form factors at zero recoil, Phys. Rev. D 61 (1999) 014502 [arXiv:hep-ph/9906376].

[39] J. M. Flynn et al., Kl3 and pion form factors using partially twisted boundary conditions, arXiv:0812.4265 [hep-lat].

[40] N. Tsutsui et al. [JLQCD Collaboration], Kaon semileptonic decay form factors in two-flavor QCD, PoS LAT2005 (2006) 357 [arXiv:hep-lat/0510068].

[41] C. Dawson, T. Izubuchi, T. Kaneko, S. Sasaki and A. Soni, Vector form factor in $K_{l 3}$ semileptonic decay with two flavors of dynamical domain-wall quarks, Phys. Rev. D 74 (2006) 114502 [arXiv:hep-ph/0607162].

[42] V. Lubicz, F. Mescia, S. Simula, C. Tarantino and f. t. E. Collaboration, $K$-> pion Semileptonic Form Factors from Two-Flavor Lattice QCD, arXiv:0906.4728 [hep-lat].

[43] P. A. Boyle et al., Kl3 semileptonic form factor from 2+1 flavour lattice QCD, Phys. Rev. Lett. 100, 141601 (2008) [arXiv:0710.5136 [hep-lat]].

[44] M. Okamoto [Fermilab Lattice and MILC Collaboration and HPQCD Collaboration], Full CKM matrix with lattice QCD, arXiv:hep-lat/0412044.

[45] J. Zanotti et. al., Determining the Kl3 form factors directly at zero momentum transfer, in proceedings of Lattice 09 POS (LAT2009) 248. 
[46] J. M. Flynn and C. T. Sachrajda [RBC Collaboration and UKQCD Collaboration], SU(2) chiral prturbation theory for Kl3 decay amplitudes, Nucl. Phys. B 812 (2009) 64 [arXiv:0809.1229 [hep-ph]].

[47] S. Aoki et al. [JLQCD Collaboration], Kaon B parameter from quenched lattice QCD, Phys. Rev. Lett. 80 (1998) 5271 [arXiv:hep-lat/9710073].

[48] Y. Nakamura, S. Aoki, Y. Taniguchi and T. Yoshie [CP-PACS Collaboration], Precise determination of $B_{K}$ and right quark masses in quenched domain-wall QCD, Phys. Rev. D 78 (2008) 034502 [arXiv:0803.2569 [hep-lat]].

[49] S. Aoki et al. [JLQCD Collaboration], $B_{K}$ with two flavors of dynamical overlap fermions, Phys. Rev. D 77 (2008) 094503 [arXiv:0801.4186 [hep-lat]].

[50] P. Dimopoulos et al., K-meson vector and tensor decay constants and BK-parameter from $N f=2$ tmQCD, PoS LATTICE2008 (2008) 271 [arXiv:0810.2443 [hep-lat]].

[51] E. Gamiz, S. Collins, C. T. H. Davies, G. P. Lepage, J. Shigemitsu and M. Wingate [HPQCD Collaboration and UKQCD Collaboration], Unquenched determination of the kaon parameter $B_{K}$ from improved staggered fermions, Phys. Rev. D 73 (2006) 114502 [arXiv:hep-lat/0603023].

[52] C. Aubin, J. Laiho and R. S. Van de Water, The neutral kaon mixing parameter $B_{K}$ from unquenched mixed-action lattice QCD, arXiv:0905.3947 [hep-lat].

[53] Y. Aoki et al., Phys. Rev. D 78, 054510 (2008) [arXiv:0712.1061 [hep-lat]]. 